( 2022 , The Authors. Published by Elsevier Inc. and Fass Inc. on behalf of the American Dairy Science Association ${ }^{\circledR}$. This is an open access article under the CC BY license (http://creativecommons.org/licenses/by/4.0/).

\title{
Use of ATP luminometry to assess the cleanliness of equipment used to collect and feed colostrum on dairy farms
}

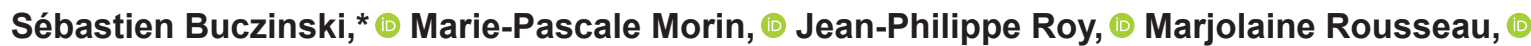 \\ Marianne Villettaz-Robichaud, $\odot$ and Jocelyn Dubuc (1) \\ Faculté de médecine vétérinaire, Université de Montréal, 3200 rue Sicotte, St-Hyacinthe, QC, J2S 2M2, Canada
}

\begin{abstract}
The objective of this observational cross-sectional study was to describe the cleanliness of various equipment used for colostrum harvest and calf feeding procedures on dairy farms in Québec, Canada. The study was performed on 42 commercial dairy herds also enrolled in another study aiming to determine the transfer of passive immunity over a 14 -mo period. Information on colostrum quality (using Brix value) and cleanliness (total aerobic and total coliform count) were recorded as well as various practices focused on colostrum-feeding equipment and preweaning period using a standard questionnaire. During the study period, colostrum and milk-feeding equipment cleanliness was assessed using direct surface swabbing with Hygiena Ultrasnap swabs. A total of 155 swab samples were obtained from 6 pieces of equipment. Adenosine triphosphate collected from the swabbed surface reacts with the luciferase solution present in the swab by bioluminescence, which is proportional to the quantity of ATP present and quantified as relative light units (RLU). The description of feed equipment cleanliness (defined as the maximal RLU found for a specific herd, dichotomized as $<1,000$ RLU vs. $\geq 1,000$ RLU) was compared with the herds' descriptive characteristics, focusing on the first 2 components of a multiple correspondence analysis. The median (range) RLU for buckets used for colostrum harvest, bucket or bottle used for feeding, tube feeders, milking colostrum line, and internal surface of the nipples were $41 \mathrm{RLU}(3-1,625 \mathrm{RLU}), 78 \mathrm{RLU}(<1-3,765$ RLU), 29 RLU ( $<1-2,177$ RLU), 83 RLU ( $<1-9,968$ RLU), and 1,101 RLU (2-9,546 RLU), respectively. The first 2 components of multiple correspondence analysis explained $24.7 \%$ of data variances and were related to the farms' hygiene and health $(13.0 \%)$ and feeding practices (11.7\% of data variance). The maxi-
\end{abstract}

Received July 16, 2021.

Accepted October 7, 2021.

*Corresponding author: s.buczinski@umontreal.ca mal dichotomized luminometry value $(<1,000$ RLU or $\geq 1,000 \mathrm{RLU}$ ) was associated with hygiene and health dimension. This study gave promising results concerning the potential application of ATP luminometry for calf rearing practices assessment.

Key words: hygiene, calf health, first-milk supply, rapid testing

\section{INTRODUCTION}

Dairy calf management is a critical component of the dairy industry because raising good quality calves is crucial for maintaining long-term herd productivity and health. Colostrum feeding is of primary importance to provide passive immunity (maternal antibodies) and other cellular and humoral components to newborn dairy calves (Puppel et al., 2019). The success of the transfer of passive immunity (TPI) can be monitored at calf and herd levels using serum refractometry on farms (Lombard et al., 2020). Monitoring the quality and cleanliness of colostrum meals is of utmost importance to ensure adequate TPI programs in dairy herds (Godden et al., 2019). Colostrum contamination is associated with decreased absorption of antibodies due to interaction between bacteria and antibodies (Godden et al., 2019). Colostrum quality can be assessed in various ways including Brix refractometry, which is a proxy for its immunoglobulin concentration (Quigley et al., 2013; Buczinski and Vandeweerd, 2016). Total aerobic and coliform counts are also negatively correlated with colostrum microbiological quality (Hyde et al., 2020). Increased bacterial counts in colostrum fed to calves can be associated with inadequate udder preparation during milking; contamination of the equipment used for collecting, storing, or delivering the meal; as well as improper storage of the colostrum before feeding (Fecteau et al., 2002; Hyde et al., 2020).

Practical and accurate on-site assessment of equipment cleanliness is a challenge on dairy farms. As recently shown on Ontario dairy farms, visual inspection of feeding equipment can lack sensitivity to detect excessively contaminated surfaces (Renaud et al., 2017). 
For this reason, some studies have focused on more objective ways to assess the hygiene of feeding and milking equipment (Renaud et al., 2017; Lindell et al., 2018). Direct swabbing or sampling of the feeding or milking equipment surfaces to quantify bacterial contamination using classic microbiological culture is generally the gold standard method (Renaud et al., 2017; Lindell et al., 2018). Determination of colostrum total bacterial counts does not enable separation between different sources of contamination, which include colostrum contamination during milking and storing as well as by contact with contaminated surfaces. Bacterial counts also give delayed results for detection of bacterial growth and some laboratory needs. A rapid assessment of ATP found on the different surfaces has been mentioned as an indirect tool for the quantification of bacterial contamination (Mildenhall and Rankin, 2020). The monitoring of ATP is already widely used as a standard approach in the medical and food industries (Mildenhall and Rankin, 2020). As such, ATP due to bacterial activity on the sampled surface is quantified using a bioluminescence assay, generally tested in a portable device (Mildenhall and Rankin, 2020). The result is displayed in relative light units (RLU) a few seconds after the swabbed material has been in contact with luciferase, an enzyme that chemically generates light.

The information available on dairy farms is scant relative to the potential use of an ATP bioluminescence assay to assess hygiene (Renaud et al., 2017). Knowing the variation of contamination levels in herds and associating it with calf-raising procedures could be helpful for dairy producers and consultants. It could help assess if the farms' equipment cleaning standard operating procedures (SOP) are adequate, and thus could help improve calf-raising programs on farms.

Therefore, the objective of this study was to describe the cleanliness of various equipment used for colostrum harvest and calf feeding procedures on Québec dairy farms. We hypothesized that excessive bacterial contamination is commonly observed on farms and that herds with excessive contamination of surfaces are different from herds with low contamination of surfaces in terms of calf and colostrum management practices.

\section{MATERIALS AND METHODS}

\section{Study Design}

The current observational cross-sectional study was part of a larger research program designed to determine the risk factors associated with successful TPI in Québec dairy herds (Morin et al., 2021a,c). The research protocol was approved by the Animal Care Committee of the Université de Montréal (16-Rech-1854). Of the
59 herds participating in the larger research program from November 2016 to January 2018, 42 herds were visited for a specific assessment of the colostrum-feeding equipment cleanliness. The initial herd population $(\mathrm{n}=$ 59) was selected conveniently among dairy farm clients from the bovine ambulatory clinic. Only a subsample could be selected based on the limited of swabs available for this study.

\section{Data Collection}

Briefly, herds were visited regularly to collect blood samples from calves of 1 to $7 \mathrm{~d}$ of age; a minimum of 14 calves per herd was sampled. Male calves were collected only if their colostrum-feeding regimens were the same in female and male calves in participating farms to avoid specific sex effect in TPI assessment (Morin et al., 2021a). The harvested blood samples were centrifuged at $1,750 \times g$ for $10 \mathrm{~min}$ at $20^{\circ} \mathrm{C}$, and sera were extracted. Serum refractive index was quantified using a digital Brix refractometer (PA203, MISCO). Inadequate TPI was defined as a serum Brix score $<8.4 \%$, which minimized misclassification errors when compared with a serum $\operatorname{IgG}<10 \mathrm{~g} / \mathrm{L}$, defined as a gold standard test (Buczinski et al., 2021). Colostrum samples were collected and frozen by participating farmers during the same period. After thawing at room temperature and mixing, the IgG content was estimated using the same Brix refractometer (PA 203, MISCO). A 1:1,000 dilution of colostrum with sterile water was then performed; $1 \mathrm{~mL}$ of the diluted solution was placed on aerobic and coliform count plates (Aerobic Count Plate and Coliform Count Plate; $3 M$ ). After incubation at $38^{\circ} \mathrm{C}$, the aerobic and coliform counts were obtained after 48 and $24 \mathrm{~h}$, respectively (Morin et al., 2021b).

A herd-level questionnaire was completed with the farmer at the beginning of the study period to know common herd characteristics and specific herd-management details about calving, colostrum management, and disease. The questionnaire contained questions on herd size (number of milking cows), total number of workers on the farm, and workers involved in calf management. The percentage of calves with inadequate TPI (defined as a serum Brix <8.4\%) was also obtained, as well as the median colostrum Brix score and aerobic and coliform counts of the colostrum. It was noted if colostrum management differed depending on calf sex. Other specific questions on colostrum management were about the presence of a frozen colostrum bank on the farm, the use of a refractometer to assess colostrum quality, the visual analysis of the first-milk strips before colostrum collection, and use of gloves during colostrum collection. The use of equipment for calf colostrum 
feeding was indicated as either a bottle with a nipple, a bucket, or tube feeder. The cleaning SOP used for colostrum equipment in different herds varied greatly. To simplify the data collection, a distinction was made between herds that did a complete equipment washing using soap after every use or not. Questions on dry cow management included the expected herd dry length period and the use of teat sealant at dry-off. Investigation of infectious disease management in the herd included questions about the general vaccination strategy used for lactating adult cows (vaccine protecting for aborting agents or respiratory diseases such as infectious bovine rhinotracheitis, para-influenza type 3 , bovine viral diarrhea virus, and bovine respiratory syncytial virus), vaccination during the dry period (vaccines for either mastitis prevention or calf diarrhea prevention), and vaccination for calves (vaccination for either respiratory disease or diarrhea). General questions were also asked on the producer's perception about the presence of respiratory disease, diarrhea, and mortality in calves and their record keeping. For these specific last 3 questions on calf health, no specific validations were performed in the absence of reliable health recording system; only the perception of the farmer on the importance of these major calves' disorders was obtained.

Each herd was visited by research staff to perform a rapid assessment of ready-to-use colostrum-feeding equipment. Possible equipment of interest included the ones for colostrum collection, storage, or distribution to calves depending on their availability. During the farm visit, up to 6 different equipment pieces were swabbed using Ultrasnap swabs (Ultrasnap, Surface ATP test, Hygiena). The swabbing technique was performed by the same operator following the SOP recommended for sampling plane surfaces by the manufacturer. Each sample was a $10 \times 10 \mathrm{~cm}$ area, which was swabbed while rotating the swab to achieve maximal contact with the sampled area. Sampled areas included the internal surface of feeding buckets, bottles, nipples, and tubes. In cases of irregular surfaces (e.g., nipples or internal surface of a feeding tube), the swab was inserted in the equipment and the surface swabbed was between the distal part of the surface and the proximal section of the surface, trying to rotate the whole surface of the swab when sweeping all the internal surface of the equipment. The Ultrasnap swab has a limit of detection of 1 femtomole/L of ATP with almost a perfect correlation between ATP concentration and RLU $(\mathrm{r}=0.98)$. Therefore, results of 0 were recorded as $<1$ RLU.

In the absence of a specific threshold to determine if colostrum-feeding equipment cleanliness was adequate or not, we considered results from 2 studies assessing milking equipment cleanliness that used thresholds from $<152$ to $<1,821$ RLU (Vilar et al., 2008) and
$<300$ RLU (Lindell et al., 2018). We also considered results from a recent review compiling 60 studies that reported thresholds for cleanliness from $\leq 1$ to $<1,000$ RLU for various medical and food contact surfaces (Mildenhall and Rankin, 2020). Based on these results and distribution of the luminometry values, we chose to use a threshold of $<1,000 \mathrm{RLU}$ to define adequate colostrum-feeding equipment cleanliness.

\section{Statistical Analysis}

All analyses were performed using the open source R software v 4.0.6 (https://www.r-project.org/). All descriptive results were reported as median and range for numeric variables. Luminometry results were reported similarly according to the sampling location. The maximum luminometry value obtained in a herd was considered representative of the hygiene level of the equipment used for colostrum management. Continuous variables that were related to colostrum meal quality [herd median colostrum Brix value, herd median aerobic and coliform counts, the proportion of inadequate TPI (i.e., proportion of calves with serum Brix <8.4\%)] and maximal luminometry value were compared using the Spearman rho coefficient. The frequency of dichotomous variables from the questionnaire was also quantified.

A multiple correspondence analysis (MCA) was performed using FactoMineR package to describe the different relationships between various categorical data in the population of individuals (Sourial et al., 2010; Husson et al., 2017). The MCA strategy was chosen because it is useful to reduce data set dimensionality and to quantify correlations between categorical variables (Husson et al., 2017). Adding supplementary variables (i.e., variables not included in MCA calculation) on the MCA visual representation can also be useful to determine the potential association between supplementary variables and variables represented in the MCA cartesian plan (Husson et al., 2017). In our analyses, the supplementary variables were luminometry value (lum_1000 as a dichotomous variable using 1,000 RLU as the cleanliness threshold) and the herd size (large vs. small based on the median number of cows). Missing data in the data set were imputed based on the imputeMCA function of the MissMDA R package, which uses regularized iterative MCA algorithms to complete the disjunctive matrix of the whole data set (Josse and Husson, 2016). As such, the MCA model ultimately presents the different dimensions in descending order from the dimension explaining the more variation of the data to the least.

The quality of MCA representation is influenced by the number of categories per item, especially when 
sample per category is low. For this reason, we used a dichotomization strategy based on the median for continuous variables ( $<$ vs. $>$ median, except for coliform counts where a median of 0 versus $>0 \mathrm{cfu} / \mathrm{mL}$ was used because of data distribution). They were then included in the MCA model as dichotomous variables.

Two supplemental variables (luminometry and number of cows) were then superposed based on their central tendency (barycenter) and 95\% confidence ellipses. These ellipses were used to compare categories within a specific variable using Factoextra package, which graphically represents MCA output (Kassambara and Mundt, 2017). This representation was used to look for the discrimination quality of supplementary variables in the different farms based on the 2 main dimensions of the MCA model. Barycenter not included in the $95 \%$ confidence ellipses of the other variable category were considered as significantly different (Husson et al., 2017).

\section{RESULTS}

A total of 42 commercial Holstein dairy farms were investigated in the current study. The median (range) number of milking cows per farm was 62 (27-275). A total of 155 luminometry swabs were collected (median: 3 samples; range: $2-5$ samples per farm). Distribution of swab contamination stratified by categories of swabbing sites is depicted in Figure 1. The median (range) RLU values for buckets used for colostrum collection, bucket or bottle, tube feeders, colostrum line, and the internal surface of the nipples were 41 RLU (3-1,625 RLU), 78 RLU (1-3,765 RLU), 29 RLU (0-2,177 RLU), 83 RLU (0-9,968 RLU), and 1,101 RLU (2-9,546 RLU), respectively. The 3 samples obtained from the teat cups used for colostrum collection were 6, 55, and 94 RLU, respectively. The distribution of maximal luminometry values in farms is represented in Figure 2. The median (interquartile range) of these data was 2,958 RLU

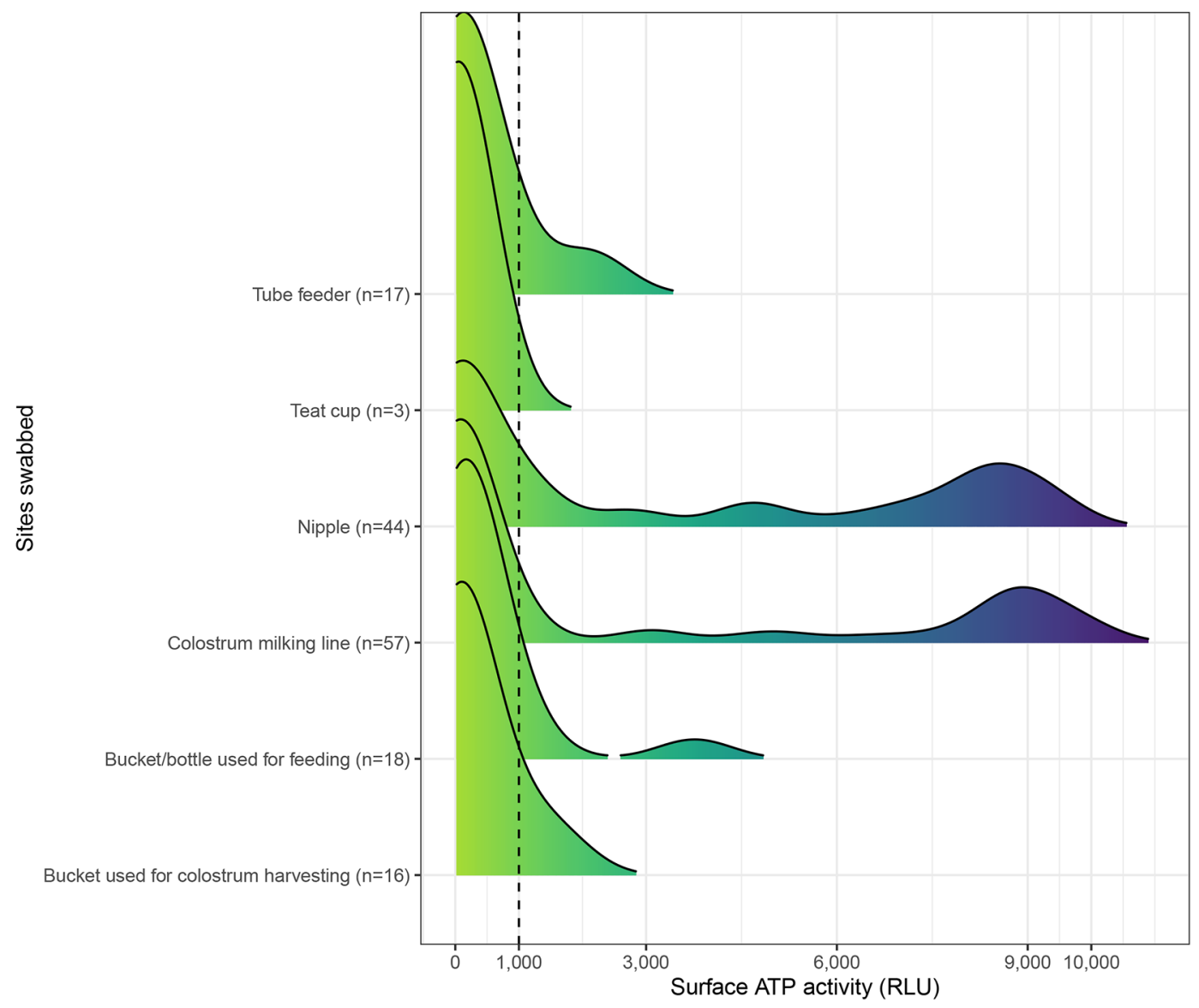

Figure 1. Distribution of luminometry measurement to assess ATP activity on the surface of various ready-to-use equipment to harvest and feed colostrum on 42 dairy farms in Québec, Canada. The ridgeline plot represents the density of distribution of luminometry measures expressed in relative light units (RLU) from various equipment. Dark colors indicate high RLU results, which are indicative of high levels of bacterial contamination. The number of samples per item is also indicated (n). The dotted line refers to the a priori selected RLU threshold ( $<1,000 \mathrm{RLU})$. The number (\%) of samples $\geq 1,000$ RLU was $0(0 \%), 24(54 \%), 19(33 \%), 2(12 \%), 2$ (13\%), and 2 (11\%) for teat cup, nipple, milk line, tube feeder, bucket used for colostrum collection, and bucket or bottle used for colostrum feeding, respectively. 
(299-8,301 RLU). The Spearman correlation rho values between the different variables assessing hygiene, colostrum quality, and percentage of inadequate TPI are depicted in Figure 3. Significant correlations were observed between maximal luminometer value and median colostrum coliform count (rho $=0.50 ; 95 \%$ CI: 0.28 to $0.71, P<0.01)$ as well as between maximal luminometer value and median colostrum aerobic count (rho $=0.50 ; 95 \%$ CI: 0.25 to $0.73, P<0.01)$. No correlation was observed between luminometry value and the proportion of inadequate TPI (rho $=0.28 ; 95 \% \mathrm{CI}$ : -0.03 to $0.60, P=0.07$ ) as well as for luminometry and median colostrum Brix (rho $=0.07 ; 95 \%$ CI: -0.30 to $0.40, P=0.65)$.

The main descriptive characteristics of the farm and farm questionnaire variables are indicated in Table 1. Almost all herds $(38 / 42 ; 90.5 \%)$ did not have a different colostrum-feeding regimen for male and female calves. The median percentage of inadequate TPI was high with a median of 30\% (range $0-70 \%$ ). The median colostrum aerobic count was $15 \times 10^{3} \mathrm{cfu} / \mathrm{mL}$, with a wide variation between $1.5 \times 10^{3}$ and $5 \times 10^{6} \mathrm{cfu} / \mathrm{mL}$. Eleven farms $(26 \%)$ had a median colostrum coliform count $>0 \mathrm{cfu} / \mathrm{mL}$. Twelve of the 39 farms $(31 \%)$ where the data were available regularly used soap for cleaning colostrum sampling and feeding equipment. Farmer

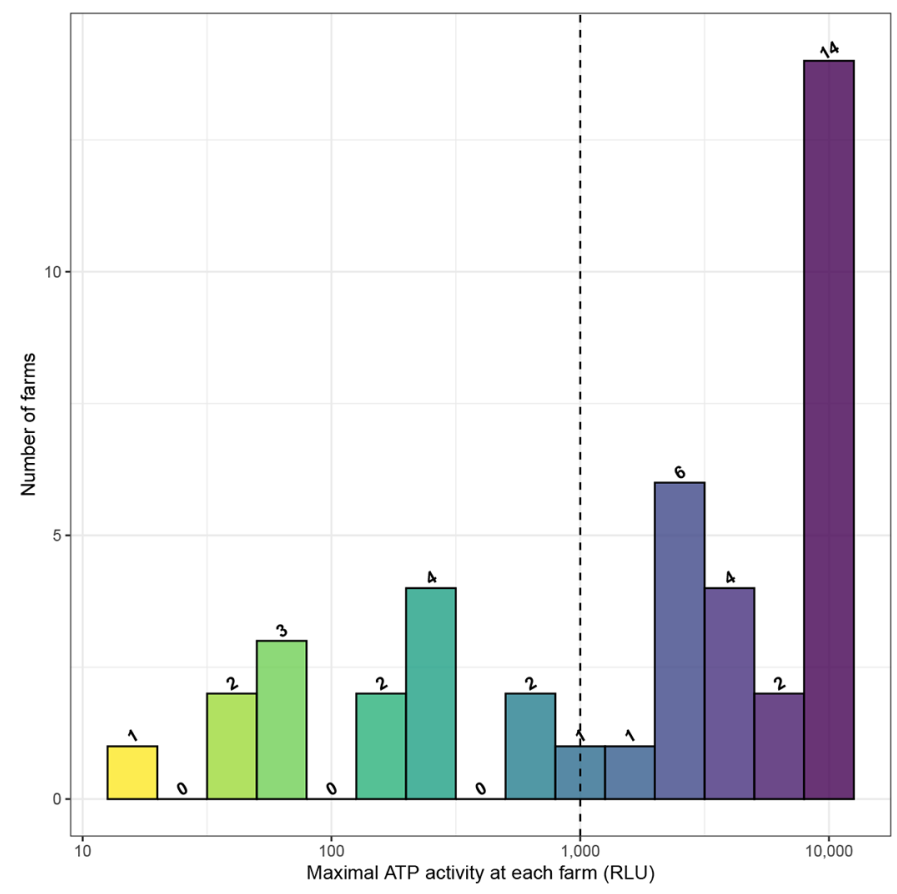

Figure 2. Distribution of luminometry measurement to assess maximal surface ATP activity in relative light units (RLU) of readyto-use equipment to harvest and feed colostrum on 42 dairy farms from Québec, Canada. The dotted line refers to the a priori selected RLU threshold $(<1,000 \mathrm{RLU})$. The number of farms per RLU strata is indicated at the top of the bars. perception of health problem revealed that diarrhea, pneumonia, and mortality were perceived as a problem in $57 \%(18 / 42), 71 \%(30 / 42)$, and $40 \%(17 / 42)$ of herds, respectively.

The MCA results are summarized by 2 main dimensions, which accounted for $13.0 \%$ and $11.7 \%$ of the variance, respectively. Based on the dimension compositions, the first one was named "hygiene and health," whereas the second one was named "feeding practices." Results of the 20 most important variables included in the MCA are indicated in Figure 4. The visual representation of the 2 dimensions allowed us to distinguish between farms with low or high proportion of inadequate TPI (dimension 1 ) and low or high median value of colostrum Brix scores (dimension 2; Figure 5). The supplementary variable representation shows that the first dimension of the MCA allowed distinction between farms having $\geq 1,000$ RLU as a maximal value of luminometry and farms with maximal values $<1,000$ RLU, whereas herd size dichotomization did not allow such discrimination on the 2-dimension plan (Figure 6).

\section{DISCUSSION}

Hygiene and sanitation procedures for milk-feeding equipment have been promoted in dairy calf-raising programs as important parts to improve calf health (Walker et al., 2012). Being able to perform a rapid assessment of farm cleaning procedures is crucial to verify if cleaning protocols are implemented correctly. The present study showed that farms with elevated bacterial and coliform counts in colostrum, high proportion of inadequate TPI, and lower health performance had higher contaminated feed equipment as investigated by swab luminometry. Despite its observational study design, our study gave interesting preliminary insights on the usefulness of this point-of-care test (luminometer) in the routine management of calves. Not surprisingly, milk lines used for colostrum harvest and internal part of nipples had the highest contamination levels of all sampled areas. The important contamination of the nipple internal surface has previously been reported in a German study (Heinemann et al., 2021). Despite using a relatively high threshold to define excessive bacterial contamination of feeding equipment ( $\geq 1,000$ RLU), $67 \%(28 / 42)$ of the visited farms had a maximal RLU value above this threshold. This may indicate that there is room for improvement in cleaning colostrum harvesting and feeding equipment. One should remember that the threshold used here was a priori defined based on review of existing literature combining evidence from different types of luminometer systems used for different purposes (Mildenhall and Rankin, 2020), including assessment of milk line equipment (Vilar et al., 2008; 
Lindell et al., 2018). Therefore, this threshold was selected based on its potential specificity (i.e., low risk of finding elevated RLU in a farm with adequate cleaning protocol). This study was not designed for establishing the optimal cut off for definition of a clean versus dirty surface. Because substantial variations may exist between luminometer types (Mildenhall and Rankin, 2020), the Hygiena 1,000 RLU threshold used cannot be extrapolated to other luminometer types.

Calves are exposed to bacteria and various pathogens during the preweaning period via their living environment and ventilation (Lago et al., 2006; Ollivett, 2020), manure, and increased contact with other animals (Lundborg et al., 2005), as well as through milk and colostrum feeding (Armengol and Fraile, 2016; Godden et al., 2019). Increased exposure to bacteria and poor hygiene are generally associated with increased risk of infectious disease (Lundborg et al., 2005; Lago et al., 2006; Klein-Jöbstl et al., 2014). Luminometry quantifies the ATP found on the surface being investigated, and it was shown to be correlated with bacterial counts (Renaud et al., 2017; Lindell et al., 2018). However, one should keep in mind that ATP does not only detect bacterial ATP. Previous studies have shown that ATP quantified using RLU values are linearly correlated with various concentrations of eukaryote cells such as red blood cells, epithelial cells, or parasites (e.g., Toxocara canis eggs or Toxoplasma gondii tachyzoites) in an in vitro study (Turner et al., 2010). Increased RLU measurement can also be associated with milk residues as previously noticed for milk liners (Lindell et al., 2018). For this reason, the RLU measurement

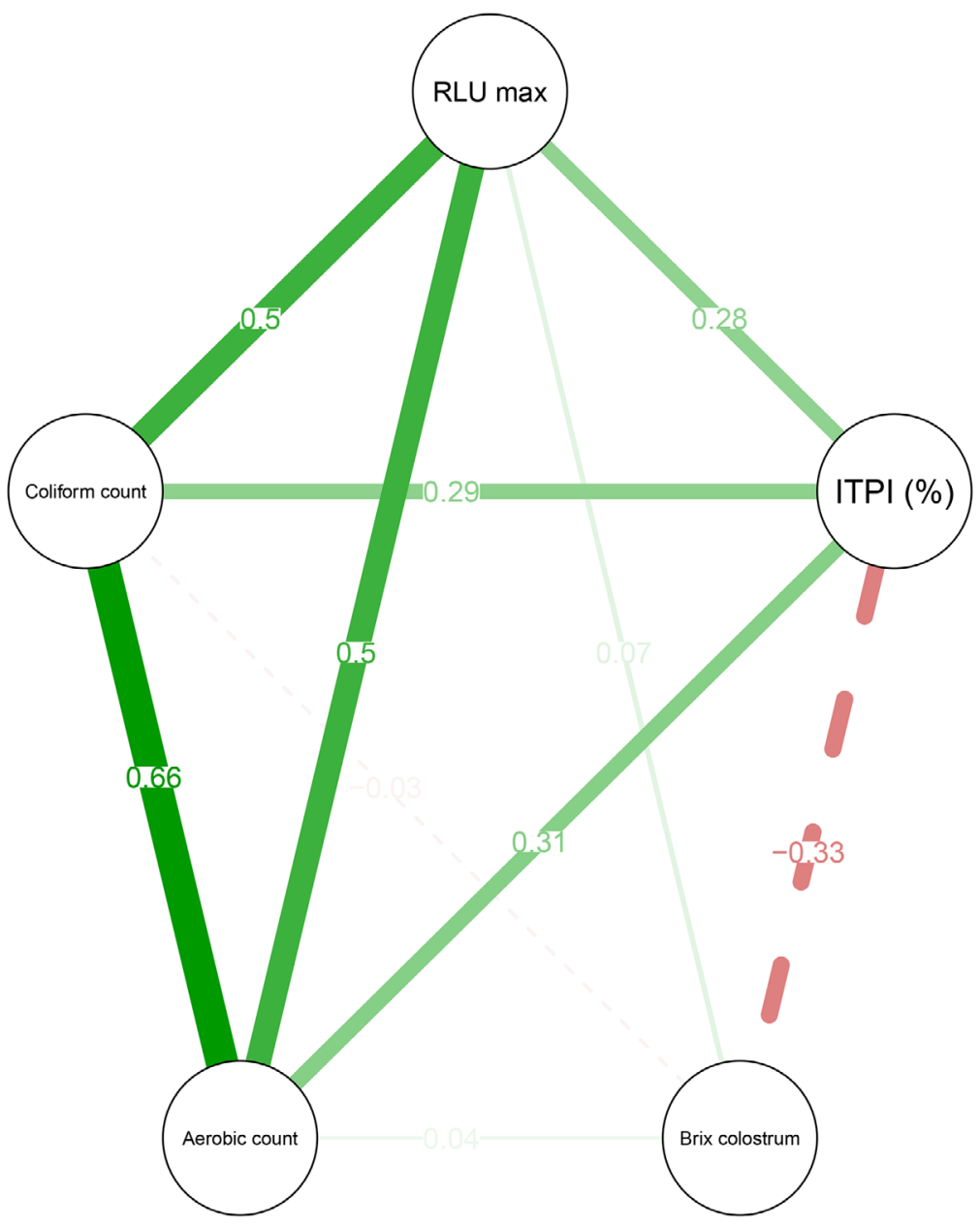

Figure 3. Correlation network between quantitative variables collected on 42 dairy farms from Québec, Canada, as indicators of colostrum quality, feeding equipment hygiene, and transfer of passive immunity. Relative light unit (RLU) max = maximal surface ATP activity collected on the farm and expressed in RLU; aerobic count = median aerobic count of colostrum samples (cfu/mL); coliform count $=$ median coliform count of colostrum samples $(\mathrm{cfu} / \mathrm{mL})$; Brix colostrum = median value of herd colostrum samples explained in \% Brix score; inadequate transfer of passive immunity (ITPI; \%) = percentage of calves with ITPI, defined as serum Brix $<8.4 \%$. The green lines indicate a positive correlation between 2 variables, whereas the red dashed lines indicate a negative correlation. The line thickness and darkness are proportional to the rho value. Nonsignificant correlations (Spearman rho with a $P>0.05)$ were aerobic count - Brix colostrum $(P=0.79)$, coliform count - Brix colostrum $(P=0.84)$, Brix colostrum - RLU max $(P=0.65)$, RLU max - ITPI $(P=0.07)$, and coliform count - ITPI $(P=0.06)$. 
Table 1. Descriptive characteristics of 42 dairy farms, including herd demographics, luminometry findings, transfer of passive immunity, ${ }^{1}$ colostrum quality, and specific management methods

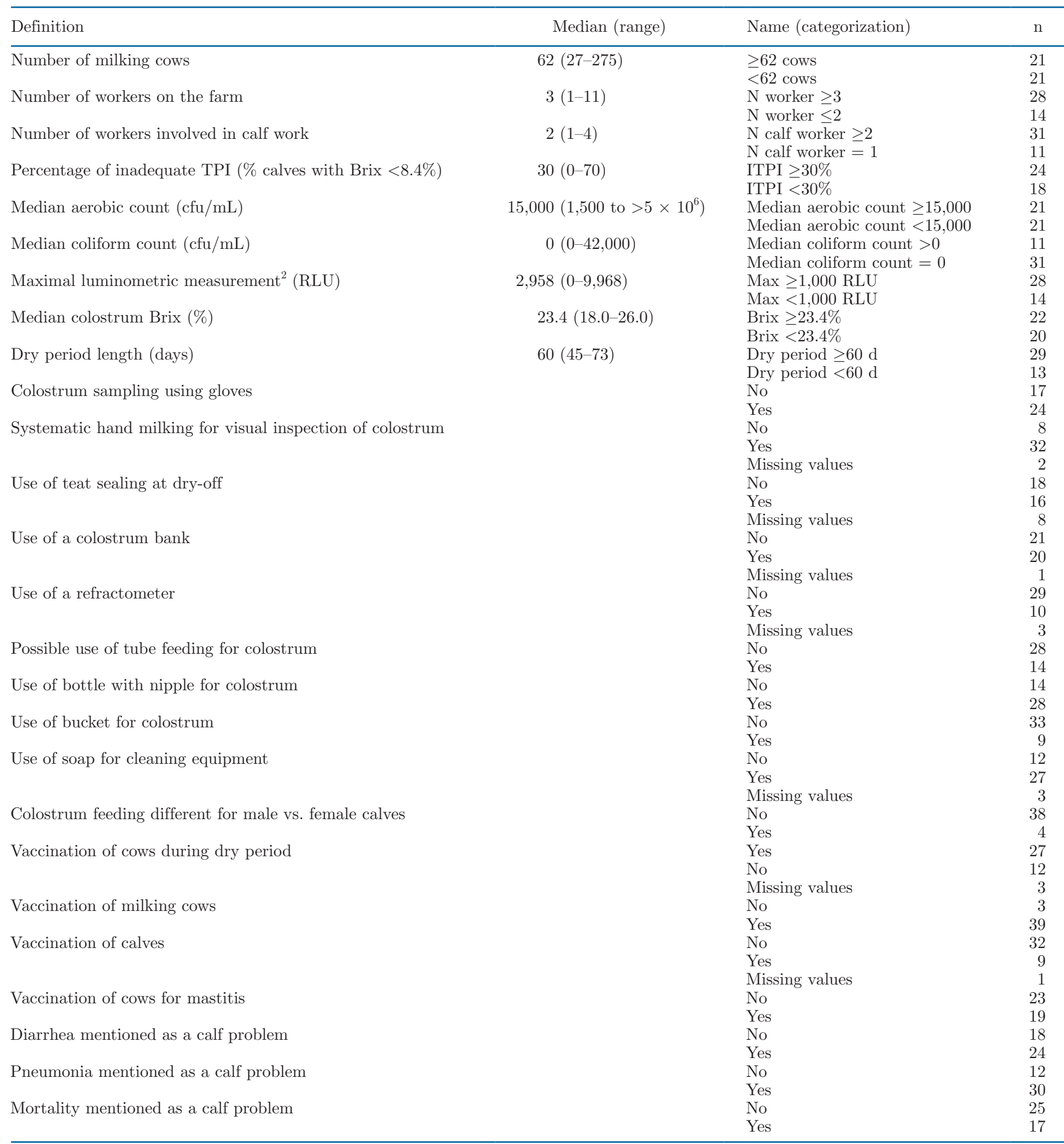

${ }^{1} \mathrm{TPI}=$ transfer of passive immunity; ITPI = inadequate TPI.

${ }^{2}$ Luminometry assesses the ATP with luciferase activity explained in terms of relative light units (RLU). 
can potentially be assumed to be a global indicator of complex interactions between microbial, eukaryote cells, and organic fragments. Therefore, it should be interpreted by keeping these limitations in mind. However, no matter what the exact causes of increased RLU values are, this still implies that cleaning and sanitizing milk-feeding equipment is not optimally performed.

Colostrum and milk-feeding equipment are especially at risk of protein, fat, and sugar deposits if improperly cleaned. These residues can serve as a matrix for future bacterial adhesion, growth, and biofilm formation, which allows future bacteria to adhere and proliferate on the contaminated surface (Teh et al., 2015). Biofilms are commonly observed in the dairy industry, especially in milking and milk-storing equipment (Teh et al., 2015). Interestingly, the correlation between luminometry values and other indicators of colostrum hygiene (i.e., median colostrum aerobic and coliform counts) was only moderate. Excessive bacterial counts in co- lostrum samples could also be increased because of inadequate teat cleaning before harvesting or inadequate storage conditions between harvesting and distribution to calves (Fecteau et al., 2002).

Only $24.7 \%$ of the data variance was explained by the 2 main dimensions of the MCA. The MCA is different from the usual principal component analysis (PCA) because it involves the use of categorical variables (e.g., $j$ variables with $k_{j}$ categories). The model is thus dependent on the complete disjunctive table evaluation and gives a higher dimensionality $\left(j \times k_{j}\right)$ than PCA analyses for the same number of variables $(j)$, explaining the higher dimensionality of the model and higher variance partitioning (Husson et al., 2017). Other components of the MCA explaining the lower part of data variance could also have been used but were considered of less importance because they only explained less than $10 \%$ of the study variance. Increasing the number of components in the presentation of the results would

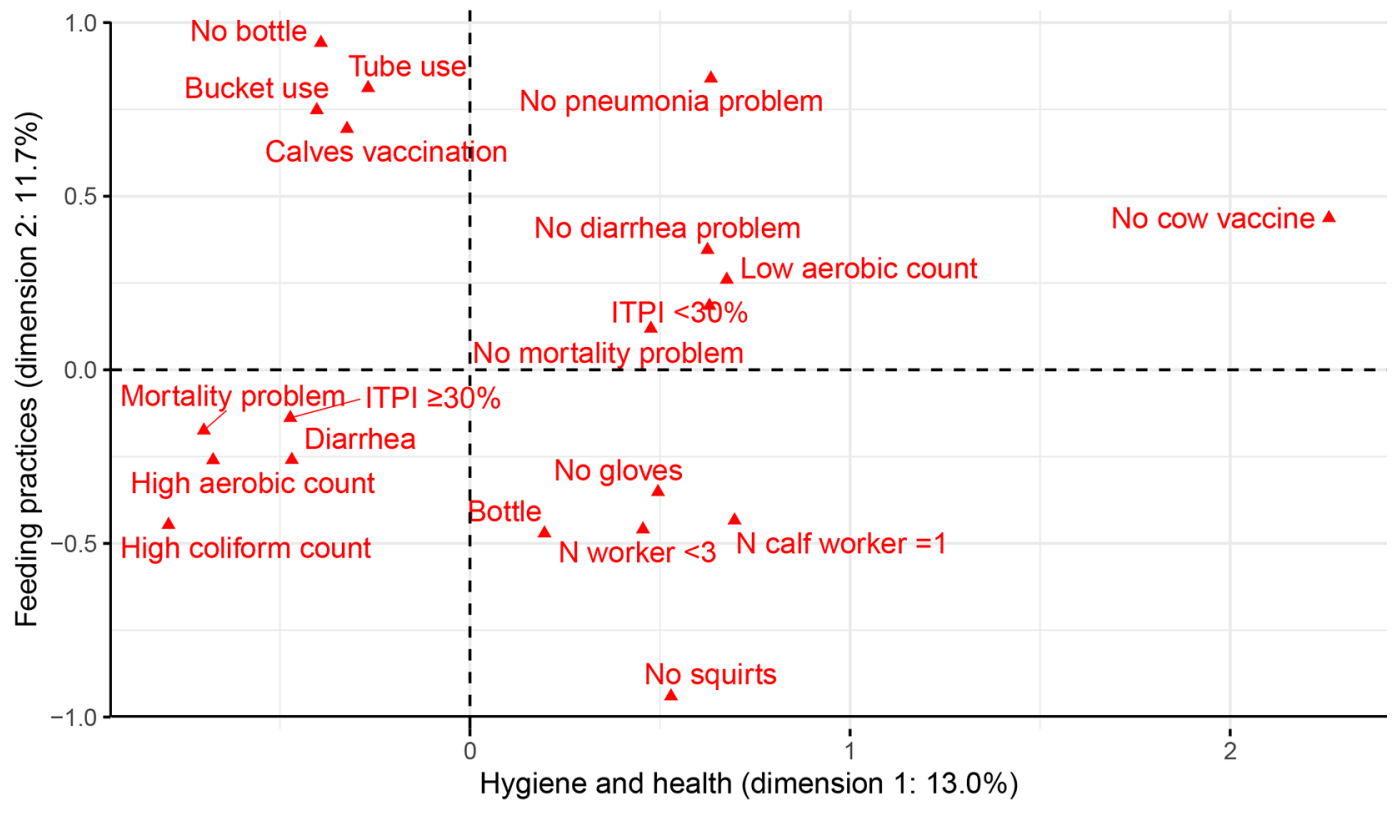

Figure 4. Graphical representation of the correlations among the 20 first variables contributing to the 2 main dimensions from the multiple correspondence analysis (MCA) summarizing herd demography, colostrum management, and calf health on 42 dairy farms from Québec, Canada. The most significant categories, explaining $23.7 \%$ of the overall data variance, are presented here. The first dimension was representative of health and hygiene categories [e.g., high median aerobic and coliform counts, and the presence of diarrhea and mortality as perceived problems on the negative pole of the x-axis, or absence of cow vaccination (no cow vaccine) on the negative pole]. The second dimension, on the y-axis, was positively associated with colostrum-feeding practice (no bottle use and the use of tube feeding or use of bucket), whereas colostrum harvesting practice that did not consider first colostrum squirts (no squirts) was represented in the negative portion of this dimension. Legends of other variables in the MCA biplot: high aerobic count = median aerobic count above the global herds median ( $\geq 15,000 \mathrm{cfu} / \mathrm{mL})$; low coliform count $=$ median aerobic count below the global herds median $(<15,000 \mathrm{cfu} / \mathrm{mL})$; no bottle = herds not using bottle with nipple for colostrum feed; bottle $=$ herds using bottle with nipple for colostrum feed; bucket $=$ use of a bucket for colostrum distribution; no mortality problem $=$ herds where mortality is not mentioned as a calf problem; mortality problem = calf mortality mentioned as a herd problem; high coliform count $=$ median coliform count $>0 \mathrm{cfu} / \mathrm{mL}$; no diarrhea problem $=$ calf diarrhea not mentioned as a herd problem; diarrhea $=$ calf diarrhea mentioned as a herd problem; ITPI $\geq 30 \%=$ proportion of calves with inadequate transfer of passive immunity $\geq 30 \%$; ITPI $<30 \%=$ proportion of calves with inadequate of transfer of passive immunity $<30 \%$; no gloves $=$ herds where no gloves are used for colostrum milking; $\mathrm{N}$ worker $<3=$ number of workers on the farm below the median $(<3)$; $\mathrm{N}$ calf worker $=1=$ number of workers taking care of calves $=1$; no pneumonia problem $=$ calf pneumonia not mentioned as a herd problem; no squirts = herds not controlling colostrum with first strips checking before collection and distribution to the calf; tube use $=$ herds that can use tube feeding for colostrum meal; calves vaccination $=$ herds that are vaccinating calves during the preweaning period; no cow vaccine $=$ no routine vaccination of the milking cows. 
also not be very helpful to interpret the data set. Even though the MCA and other PCA-like analyses have many advantages, one should keep in mind that they are mostly useful for describing complex relationships within large data sets.

The present study comes with limitations. Sampled farms were selected based on their willingness to participate, which can limit the external validity of our results; motivated farmers could have different farm characteristics than the average Québec dairy farm. However, the wide range of inadequate TPI prevalence in the recruited herds showed that these herds did not have highly efficient TPI protocols. The specific health variables were based on producers' own perceptions of the problem in the absence of reliable and objective health records, which makes these variables at risk of bias due to specific farmer perception as previously noted in the 2018 Canadian national dairy study on heifer management for mortality data (Winder et al., 2018). However, these variables were generally well represented in the first MCA dimension, which was in accordance with their biological meaning. Further studies that more objectively monitor these parameters should be conducted to specify potential association with feeding equipment device cleanliness. Considering our methodology, the determination of hygiene status on farms was based on punctual and infrequent testing. It remains unknown if maximal surface contamination remains constant over time. In a previous study, German researchers found limited improvement of luminometry measurement in 11 dairy farms monitored 1 yr apart. This finding was not expected because they anticipated that participation in the initial sampling period and reinforcement of cleaning strategies would have a longterm positive effect (Heinemann et al., 2021).

The type of equipment sampled during our study depended on what was available at the time of the farm visit. The specific protocol used in the farm was to sample only previously cleaned and ready-to-use equipment, which may decrease the possible number of sites to sample during the visit. Moreover, due to the complexity and various shapes of the equipment sampled, validation of a specific protocol to swab these different types of surfaces will need to be further explored. The

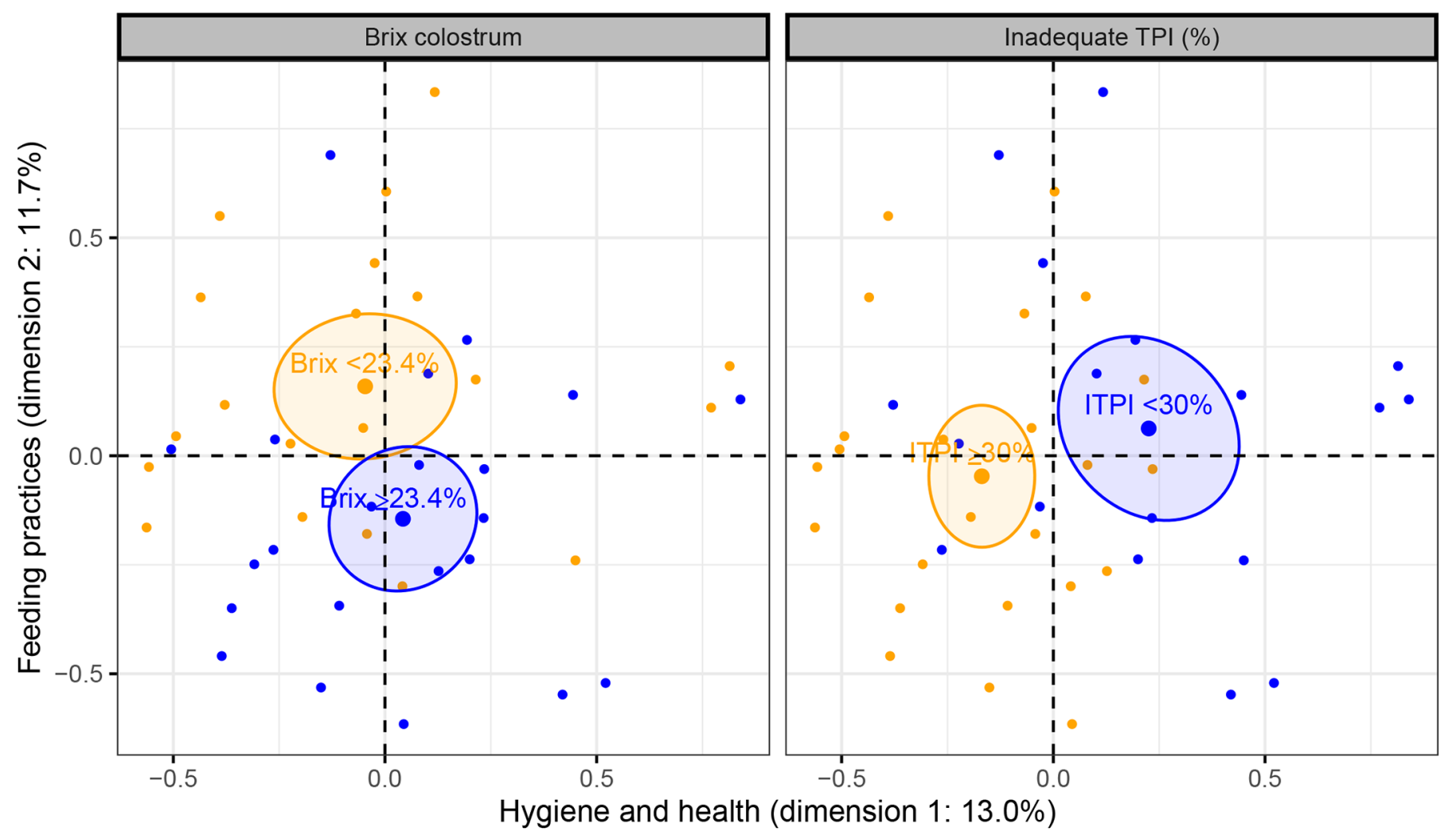

Figure 5. Representation of farms with low or high median Brix colostrum values and of farms with low percentage of inadequate of transfer of passive immunity (ITPI) based on multiple correspondence analyses (MCA) summarizing herd demography, colostrum management, and calf health on 42 dairy farms from Québec, Canada. Each dot represents a farm on the 2 main dimensions of the MCA analysis. The color of each dot depends on the response to 2 dichotomous variables involved in MCA. The barycenter (larger point) and its associated $95 \%$ confidence interval ellipses are presented. The second dimension (y-axis) allowed discrimination of the herds based on their median Brix levels, whereas the transfer of passive immunity (TPI) was used to discriminate farms on the first dimension of the MCA. 


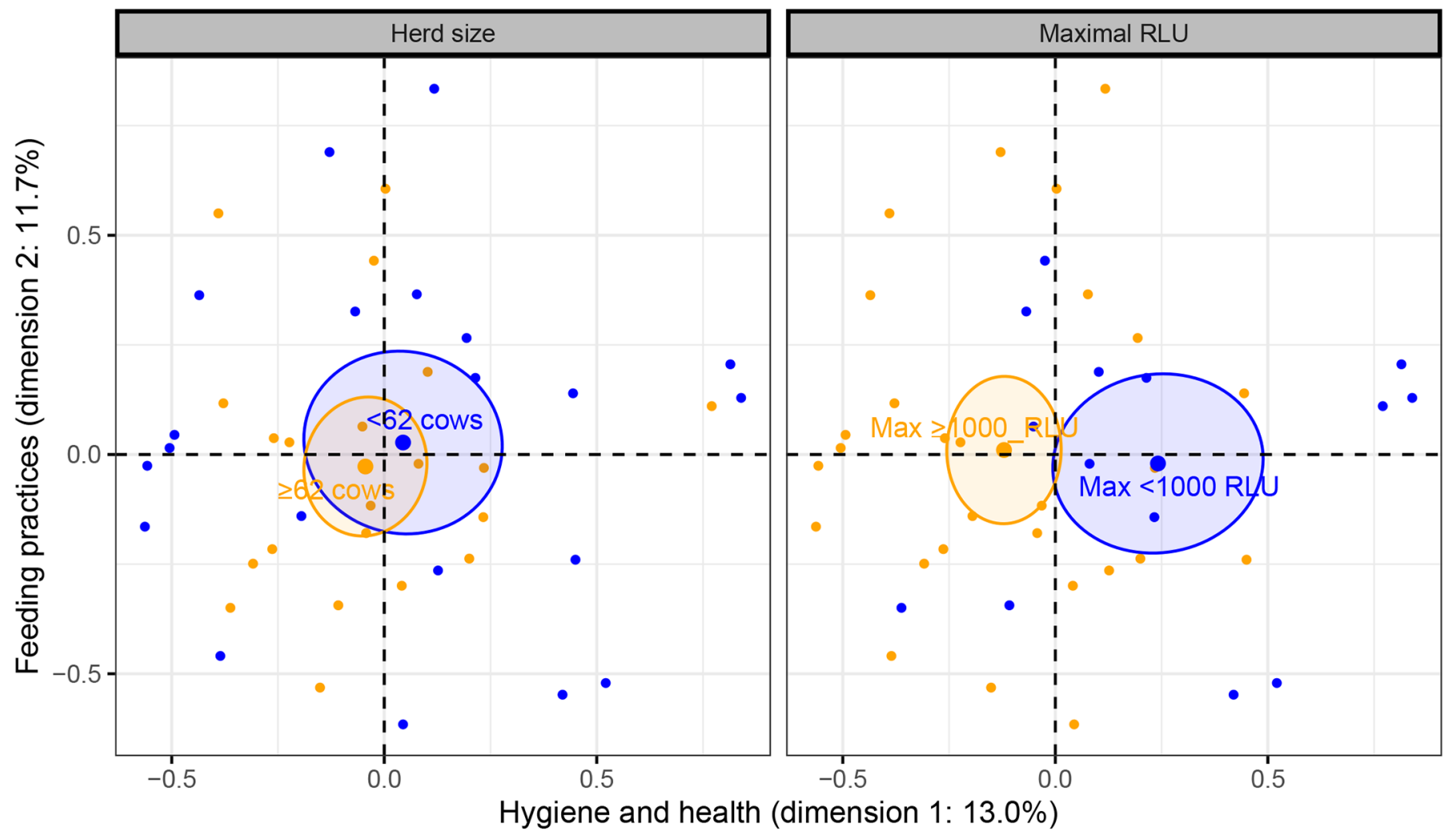

Figure 6. Representation of the 2 supplementary variables (herd size and maximal luminometry values) on the main 2-dimensional plan from multiple correspondence analyses (MCA) to summarize herd demography, colostrum management, and calf health on 42 dairy farms from Québec, Canada. The 2 supplementary variables are represented. The variable herd size represents farms where $\geq 62$ milking cows were present versus farms with $<62$ milking cows. The variable maximal relative light unit (RLU) represents herds with maximal luminometry value $<1,000$ RLU or $\geq 1,000 \mathrm{RLU}$ for ready-to-use colostrum-feeding equipment. The dichotomous variables are represented depending on their barycenter and $95 \%$ confidence ellipse. The 42 farms are displayed using colors associated with their value. The herd size was not discriminated, as shown by the wide overlapping of the 95th confidence interval ellipses. Farms with high maximal luminometry were discriminated from herds having low luminometry values on the first dimension (x-axis), as shown with point-estimate barycenters not included in the 95th confidence interval ellipse.

current study could serve as a proof-of-concept study that needs to be followed by larger studies. Previous studies have shown that luminometry is very useful to detect cleanliness problems in apparently clean materials. Because luminometry can be performed rapidly on site and that it is an objective measurement, it can potentially be implemented in dairy farms to determine the global washing and sanitation management in the farms as it is implemented in various agroalimentary sectors (Mildenhall and Rankin, 2020). An indirect suggestion from the current study would be to use luminometry as a quick screening test for the investigation of global hygiene colostrum management. However, due to the observational nature of the study and the punctual assessment of hygiene with luminometry, this should be confirmed by future studies.

\section{CONCLUSIONS}

This observational study showed that the cleanliness of equipment used for collecting and feeding colostrum when punctually assessed by luminometry was useful to distinguish dairy herds according to other management practices and hygiene characteristics. Considering the current results, we think that the use of a portative luminometer device is promising as a quick on-site tool to help check farm SOP for colostrum and milk-feeding equipment cleaning and sanitation practices. Future research should determine the optimal way for tailoring the use of luminometer in calf barns as well as on the positive effect of decreasing luminometry values on calf health and performance.

\section{ACKNOWLEDGMENTS}

This project was a specific addendum to a larger project supported by Zoetis Clinical Research Fund of the Bovine Ambulatory Clinic of the Faculté de médecine vétérinaire of the Université de Montréal (St-Hyacinthe, QC, Canada). This study was financially supported by the Fondation du Centre Hospitalier Universitaire Vétérinaire (CHUV; St-Hyacinthe, QC, Canada) of 
the Université de Montréal. The authors thank Guy Boisclair and Merck Animal Health for providing the luminometer and parts of the swabs to perform the analyses. The authors also thank the farmers for their participation in the study and Jean-Philippe Pelletier (St-Hyacinthe, QC, Canada) for his technical support. The authors have not stated any conflicts of interest.

\section{REFERENCES}

Armengol, R., and L. Fraile. 2016. Colostrum and milk pasteurization improve health status and decrease mortality in neonatal calves receiving appropriate colostrum ingestion. J. Dairy Sci. 99:47184725. https://doi.org/10.3168/jds.2015-10728.

Buczinski, S., Y. Lu, M. Chigerwe, G. Fecteau, and N. Dendukuri. 2021. Systematic review and meta-analysis of refractometry for diagnosis of inadequate transfer of passive immunity in dairy calves: Quantifying how accuracy varies with threshold using a Bayesian approach. Prev. Vet. Med. 189:105306. https://doi.org/10.1016/j .prevetmed.2021.105306.

Buczinski, S., and J. M. Vandeweerd. 2016. Diagnostic accuracy of refractometry for assessing bovine colostrum quality: A systematic review and meta-analysis. J. Dairy Sci. 99:7381-7394. https://doi .org/10.3168/jds.2016-10955.

Fecteau, G., P. Baillargeon, R. Higgins, J. Paré, and M. Fortin. 2002. Bacterial contamination of colostrum fed to newborn calves in Québec dairy herds. Can. Vet. J. 43:523-527.

Godden, S. M., J. E. Lombard, and A. R. Woolums. 2019. Colostrum Management for Dairy Calves. Vet. Clin. North Am. Food Anim. Pract. 35:535-556. https://doi.org/10.1016/j.cvfa.2019.07.005.

Heinemann, C., C. D. Leubner, J. J. Hayer, and J. Steinhoff-Wagner. 2021. Hygiene management in newborn individually housed dairy calves focusing on housing and feeding practices. J. Anim. Sci. 99:skaa391.

Husson, F., S. Lê, and J. Pagès. 2017. Exploratory multivariate analysis by example using R. CRC press.

Hyde, R. M., M. J. Green, C. Hudson, and P. M. Down. 2020. Quantitative analysis of colostrum bacteriology on British dairy farms. Front. Vet. Sci. 7:601227. https://doi.org/10.3389/fvets.2020 601227.

Josse, J., and F. Husson. 2016. missMDA: A package for handling missing values in multivariate data analysis. J. Stat. Softw. 70:131. https://doi.org/10.18637/jss.v070.i01.

Kassambara, A., and F. J. E. Mundt. 2017. Package 'factoextra'. 76. https://cran.r-project.org/web/packages/factoextra/index.html.

Klein-Jöbstl, D., M. Iwersen, and M. Drillich. 2014. Farm characteristics and calf management practices on dairy farms with and without diarrhea: A case-control study to investigate risk factors for calf diarrhea. J. Dairy Sci. 97:5110-5119. https://doi.org/10.3168/ jds.2013-7695.

Lago, A., S. M. McGuirk, T. B. Bennett, N. B. Cook, and K. V. Nordlund. 2006. Calf respiratory disease and pen microenvironments in naturally ventilated calf barns in winter. J. Dairy Sci. 89:40144025. https://doi.org/10.3168/jds.S0022-0302(06)72445-6.

Lindell, I. C., A. Lundh, K. S. Sjaunja, and M. Cederholm. 2018. Adenosine triphosphate bioluminescence for hygiene testing of rubber liners and tubes on dairy farms. J. Dairy Sci. 101:2438-2447. https: //doi.org/10.3168/jds.2017-13466.

Lombard, J., N. Urie, F. Garry, S. Godden, J. Quigley, T. Earleywine, S. McGuirk, D. Moore, M. Branan, M. Chamorro, G. Smith, C. Shivley, D. Catherman, D. Haines, A. J. Heinrichs, R. James, J. Maas, and K. Sterner. 2020. Consensus recommendations on calf-and herd-level passive immunity in dairy calves in the United States. J. Dairy Sci. 103:7611-7624. https://doi.org/10.3168/jds 2019-17955.

Lundborg, G. K., E. C. Svensson, and P. A. Oltenacu. 2005. Herd-level risk factors for infectious diseases in Swedish dairy calves aged
0-90 days. Prev. Vet. Med. 68:123-143. https://doi.org/10.1016/j .prevetmed.2004.11.014.

Mildenhall, K. B., and S. A. Rankin. 2020. Implications of adenylate metabolism in hygiene assessment: A review. J. Food Prot. 83:1619-1631. https://doi.org/10.4315/JFP-20-087.

Morin, M. P., J. Dubuc, P. Freycon, and S. Buczinski. 2021a. A calflevel study on colostrum management practices associated with adequate transfer of passive immunity in Québec dairy herds. J. Dairy Sci. 104:4904-4913. https://doi.org/10.3168/jds.2020-19475.

Morin, M. P., J. Dubuc, P. Freycon, and S. Buczinski. 2021b. Diagnostic accuracy of the Petrifilm culture system for identifying colostrum with excessive bacterial contamination in Quebec dairy herds. J. Dairy Sci. 104:4923-4928. https://doi.org/10.3168/jds .2020-19474.

Morin, M. P., J. Dubuc, P. Freycon, and S. Buczinski. 2021c. A herdlevel study on colostrum management factors associated with the prevalence of adequate transfer of passive immunity in Québec dairy herds. J. Dairy Sci. 104:4914-4922. https://doi.org/10.3168/ jds.2020-19476.

Ollivett, T. L. 2020. How does housing influence bovine respiratory disease in dairy and veal calves? Vet. Clin. North Am. Food Anim. Pract. 36:385-398. https://doi.org/10.1016/j.cvfa.2020.03.012.

Puppel, K., M. Gołębiewski, G. Grodkowski, J. Slósarz, M. KunowskaSlósarz, P. Solarczyk, M. Łukasiewicz, M. Balcerak, and T. Przysucha. 2019. Composition and factors affecting quality of bovine colostrum: A review. Animals (Basel) 9:1070. https://doi.org/10 $.3390 /$ ani9121070.

Quigley, J. D., A. Lago, C. Chapman, P. Erickson, and J. Polo. 2013. Evaluation of the Brix refractometer to estimate immunoglobulin G concentration in bovine colostrum. J. Dairy Sci. 96:1148-1155. https://doi.org/10.3168/jds.2012-5823.

Renaud, D. L., D. F. Kelton, S. J. LeBlanc, D. B. Haley, A. B. Jalbert, and T. F. Duffield. 2017. Validation of commercial luminometry swabs for total bacteria and coliform counts in colostrum-feeding equipment. J. Dairy Sci. 100:9459-9465. https://doi.org/10.3168/ jds.2017-13228.

Sourial, N., C. Wolfson, B. Zhu, J. Quail, J. Fletcher, S. Karunananthan, K. Bandeen-Roche, F. Béland, and H. Bergman. 2010. Correspondence analysis is a useful tool to uncover the relationships among categorical variables. J. Clin. Epidemiol. 63:638-646. https: //doi.org/10.1016/j.jclinepi.2009.08.008.

Teh, K. H., S. Flint, J. Brooks, and G. Knight. 2015. Biofilms in the dairy industry. John Wiley \& Sons.

Turner, D. E., E. K. Daugherity, C. Altier, and K. J. Maurer. 2010. Efficacy and limitations of an ATP-based monitoring system. J. Am. Assoc. Lab. Anim. Sci. 49:190-195.

Vilar, M. J., J. L. Rodríguez-Otero, F. Diéguez, M. Sanjuán, and E. Yus. 2008. Application of ATP bioluminescence for evaluation of surface cleanliness of milking equipment. Int. J. Food Microbiol. 125:357-361. https://doi.org/10.1016/j.ijfoodmicro.2008.04.024.

Walker, W. L., W. B. Epperson, T. E. Wittum, L. K. Lord, P. J. Rajala-Schultz, and J. Lakritz. 2012. Characteristics of dairy calf ranches: morbidity, mortality, antibiotic use practices, and biosecurity and biocontainment practices. J. Dairy Sci. 95:2204-2214. https://doi.org/10.3168/jds.2011-4727.

Winder, C. B., C. A. Bauman, T. F. Duffield, H. W. Barkema, G. P. Keefe, J. Dubuc, F. Uehlinger, and D. F. Kelton. 2018. Canadian national dairy study: Heifer calf management. J. Dairy Sci. 101:10565-10579. https://doi.org/10.3168/jds.2018-14680.

\section{ORCIDS}

Sébastien Buczinski $\odot$ https://orcid.org/0000-0002-8460-4885 Marie-Pascale Morin ( https://orcid.org/0000-0002-3671-0130 Jean-Philippe Roy @ https://orcid.org/0000-0002-0444-2303 Marjolaine Rousseau @ https://orcid.org/0000-0002-2702-434X Marianne Villettaz-Robichaud (๑) https://orcid.org/0000-0001-9685 $-1827$

Jocelyn Dubuc ํ https://orcid.org/0000-0003-1013-8372 